\title{
DECADAL CLIMATE PREDICTABILITY AND PREDICTION Where Are We?
}

\author{
by Vikram Mehta, Gerald Meehl, Lisa Goddard, Jeff Knight, Arun Kumar, \\ Mojib Latif, Tong Lee, Anthony Rosati, and Detlef Stammer
}

T he importance of decadal climate variability (DCV) research is being increasingly recognized, including by the World Climate Research Program (WCRP) and the Intergovernmental Panel on Climate Change (IPCC). An improved understanding of DCV is very important because stakeholders and policymakers want to know the likely climate trajectory for the coming decades for applications to water resources, agriculture, energy, and infrastructure development. Responding to this demand, many climate modeling groups in the United States, Europe, Japan, and elsewhere are gearing up to assess the potential for decadal climate predictions. The magnitudes of regional DCV often exceed those associated with the trends resulting from anthropogenic changes. Therefore, differentiating between the two is also very important for planning, implementation, and national and international treaties.

This brief meeting summary (presentations available at www.DecVar.org/auditorium.php) provides an overview of the consensus of a research community workshop ${ }^{1}$ on DCV held in the fall of 2009. The consensus of the participants was that decadal climate predictions may become useful to society some time in the future, but will be highly experimental in the

\section{THE EIGHTH WORKSHOP ON DECADAL}

\section{CLIMATE VARIABILITY}

WHAT: Forty-five researchers met to discuss strategies to better understand and predict decadal climate variability.

WHEN: 12-15 October 2009

Where: St. Michaels, Maryland

near future. Reflecting the increasing interest in decadal climate predictions since the previous DCV Workshop in May 2007, the main theme this time was "Decadal climate predictability and prediction: Where are we?"; the purpose was to describe and summarize the status of decadal climate nowcasting and forecasting, thus outlining a research strategy to secure the needed observations and methods to quantify predictability of DCV, and to identify unsolved science problems. Gaps in our knowledge of DCV were identified, as were areas of needed research. Recommendations in areas of predictability and prediction; observations, theory, and modeling; and societal impacts are summarized. Highest-priority

${ }^{1}$ The workshop was sponsored by the National Aeronautics and Space Administration (NASA) Physical Oceanography Program, the National Science Foundation Climate and Large-Scale Dynamics Program, the Department of Energy Office of Biological and Environmental Research, the International Climate Variability and Predictability (CLIVAR) project of the World Climate Research Programme (WCRP), and the U.S. CLIVAR Program. 
recommendations for progress toward producing useful predictions are outlined in the sidebar "Highest priorities for DCV research.”

PREDICTABILITY AND PREDICTION. Initial decadal prediction efforts in the last few years show predictive skill of global average temperature up to a decade in advance using both initial conditions and the climate change signal created by already emitted greenhouse gases. Many of the recent decadal predictability and prediction studies focused on the North Atlantic region. Idealized studies, or the so-called perfect-model experiments that do not use observations in the model initialization, suggest that some states of the Atlantic meridional overturning circulation (AMOC), such as extremes and transitions from a weak to a strong state, may be more predictable. Results also suggest that convection in the Labrador Sea governs decadal-multidecadal AMOC variability and predictability. The analysis also demonstrates the essential role of the Argo network in realizing the AMOC predictability, raising questions about the use of historic ocean observations to validate and initialize predictions. Initial experiments also show that the initialization of decadal climate predictions by three-dimensional (3D) temperature and salinity fields may provide skill in some regions up to a decade in advance, particularly over the North Atlantic and North Pacific Oceans. These initial predictability studies also suggest that there may be very small skill over land, that extratropical regions may be more predictable than tropical regions, and that there may be some skill in the predictability of extreme weather event statistics.

AfFILIATIONS: MeHTA-Center for Research on the Changing Earth System, Clarksville, Maryland; MeeHL—NCAR, Boulder, Colorado; GoDDARD - International Research Institute for Climate and Society, Palisades, New York; KNIGHT-Met Office Hadley Centre, Exeter, United Kingdom; Kumar-NOAA/ Climate Prediction Center, Camp Springs, Maryland; LATIF-IFMGEOMAR, Kiel, Germany; LeE—NASA Jet Propulsion Laboratory, Pasadena, California; RosATI-NOAA/Geophysical Fluid Dynamics Laboratory, Princeton, New Jersey; STAMMER-University of Hamburg, Hamburg, Germany

CORRESPONDING AUTHOR: Vikram Mehta, P.O. Box 346, Center for Research on the Changing Earth System, Clarksville, MD 21029

E-mail:vikram@crces.org

DOI:10.1175/20IOBAMS3025.I

In final form 27 September 2010

(C)2011 American Meteorological Society
Based on initial predictability and prediction studies, workshop participants identified the following requirements for decadal climate predictions: 1) better characterization and mechanistic understanding of decadal climate variability; 2) sustained global ocean observations; 3 ) advanced assimilation and initialization systems; 4) models with much higher resolutions to improve the representation of climate variability and reduce model biases, and accurate representation of processes such as oceanic overturning circulations, clouds, and cloud-radiation-aerosol interactions; 5) more accurate estimates of future changes in radiative forcing; 6) larger sets of nowcasts/hindcasts for the instrumental record period; 7) coordinated efforts to identify the best practice to initialize decadal predictions using ocean or coupled ocean-atmosphere data assimilation products; 8 ) systematic examination of the predictability of decadal signals in different coupled models; and 9) a better understanding of sources of potential predictability.

The most important gaps in our knowledge of predictability and prediction can be summed up in the following questions: a) How can the coupled initialization problem be solved if systematic errors in the atmosphere component of a coupled model introduce errors in the ocean component that affect hindcasts/forecasts? b) How can we nowcast DCV in the presence of higher-frequency variability? c) How do we evaluate hindcasts with respect to a changing ocean observing system? d) How can we quantify the current state of multidecadal phenomena such as the Pacific decadal oscillation (PDO) and the Atlantic multidecadal oscillation (AMO)?

OBSERVATIONS. As described and discussed in previous workshops, the quality of observed ocean data is a major concern, as are the changes in coverage resulting from changes in observing systems. The lack of salinity data before the deployment of Argo floats hampers our ability to describe DCV and verify its hindcasts because the salinity influence on density plays an important role in the oceanic components of DCV. Also, data from the Argo network has increased AMOC predictability compared to data from the expendable bathythermograph (XBT) network. This raises questions about using historical data to validate predictions. Satellite data in the past two decades (e.g., altimeters and scatterometers) have demonstrated their importance in providing a global view of DCV and insight into the ocean's role in DCV. Much of our future progress hinges on the continuity of these satellite missions and the ability to produce climate 
data records. In view of the above, the workshop participants made the following recommendations: 1) monitoring $A M O C$ using subsurface and surface measurements emphasizes the need for satellites and the Argo network, and therefore these observing systems should be continued; 2) the use of highresolution paleoclimate proxy data should be expanded because the short observational record and model uncertainty are unable to simulate DCV; and 3) until a demonstrably sufficient observing system is implemented, it is of critical importance to enhance the existing observing systems, including Argo, for decadal climate prediction.

THEORY AND MODELING. Although global coupled models designed in the last 15 years are able to generate DCV patterns that resemble observed DCV patterns, the models tend to displace them spatially and temporally with respect to observed patterns. Also, it has not been obvious that the same mechanisms operate in both models and nature to produce similar DCV patterns. A much better understanding of the physical mechanisms of DCV in nature is required. Without this, the sources and skills used to make decadal predictions will remain unreliable.

Among the known, major problems in global coupled models are large systematic biases; the absence of eddies and nonlinear interactions in ocean components; incorrect/inaccurate representation of planetary wave dynamics, interactions with eddies, $3 \mathrm{D}$ basin modes, and forced responses of basin modes; air-sea interaction; representation of vertical mixing in the upper ocean; and subpolar ocean dynamics, including the relative importance of temperature, salinity, wind-driven and thermohaline circulations, weak vertical stratification, and interactions with sea ice. The atmospheric components of the global coupled models are also not complete; the most important required additions are a well-resolved stratosphere that includes its chemical makeup, the representation of ice in the water cycle, and a better parameterization of convection, cloud physics, and tropospheric chemistry. Because resolution appears to be one of the model attributes influencing DCV time scales, model resolution is another aspect that needs major improvement. Major biases, however, are not removed simply by increasing resolution; persistent problems such as poor representations of the Indian summer monsoon rainfall still remain even in highresolution models.

With respect to these concerns, the workshop participants recommended the following: 1) there

\section{HIGHEST PRIORITIES FOR DCV}

\section{RESEARCH}

- More accurate characterization, better mechanistic understanding, and identification of sources of potential predictability of DCV are necessary.

- It is of critical importance to not only maintain but also to enhance the existing observing systems, including Argo, for decadal climate prediction.

- Reduce model errors by increasing resolution and by other measures; this requires an order of magnitude or larger increase in computing power and sustained programs by model development centers to identify, understand, and correct principal model errors.

- Elevate societal usefulness of decadal climate predictions.

is an urgent need to be consistent with terminology for DCV phenomena; for example, it is more appropriate to use "variability" than "oscillation"; 2) theoretical studies for understanding the role of ocean dynamics in DCV should be strongly supported; 3) the robustness of DCV in coupled models should be investigated systematically, especially with respect to model attributes such as resolution; 4) it is important to communicate to the scientific community, stakeholders, policymakers, and the general public the similarities and differences between natural climate variability and anthropogenic climate changes; 5 ) it is very important to separate natural DCV from anthropogenic climate changes in observations and model simulations/predictions/projections, and to better constrain climate sensitivity (and, hence, climate projections), so that both can be used for decadal climate predictions; 6) observations have revealed important features of decadal variability of ocean circulations as well as their relationship (the causes and consequences of these need further investigation); and 7) development of improved models should be strongly supported and the computing resources needed for such models should be provided.

SOCIETAL IMPACTS. In view of formal and informal concerns expressed by workshop participants about who might use DCV information, including decadal climate predictions, and what kind of information might be required by the users, workshop participants recommended the following: 1) there is a very urgent need to compile information about the social sectors impacted by DCV, the types of DCV information required for decision 
making, and the role of DCV information in the decision-making process; 2) user requirements must be sector specific and should include the extent to which they can use probabilistic DCV information; 3) societal impacts of DCV nowcasts and forecasts should be ascertained; 4) societally relevant indices of DCV phenomena, such as upwelling to help fishery management, should be identified; 5) the societal relevance aspect should be enhanced in decadal variability and predictability/prediction studies; and 6) DCV nowcasts and forecasts should be made relevant to impacted sectors, such as the DCV influ- ence on high-frequency weather variability, which is important to agriculture.

ACKNOWLEDGMENTS. The Organizing Committee is grateful to Eric Lindstrom, NASA Physical Oceanography Program; Jay Fein, NSF Climate and Large-scale Dynamics Program; and Anjuli Bamzai, the Department of Energy Office of Biological and Environmental Research for their support and encouragement for this workshop. Insightful comments by Noel Keenlyside on an earlier version of this report are also gratefully acknowledged. 